\title{
On the structural and electronic properties of Ir-silicide nanowires on $\mathrm{Si}(001)$ surface
}

\author{
Fatima, ${ }^{1}$ Ismail Can Oguz, ${ }^{2}$ Deniz Cakır ${ }^{3}$ Sehtab Hossain, ${ }^{1}$ Rasika Mohottige, ${ }^{1}$ \\ Oguz Gulseren, ${ }^{2, a)}$ and Nuri Oncel ${ }^{1, a)}$ \\ ${ }^{1}$ Department of Physics and Astrophysics, University of North Dakota, Grand Forks, North Dakota 58202, \\ USA \\ ${ }^{2}$ Department of Physics, Bilkent University, Ankara, Turkey \\ ${ }^{3}$ Department of Physics, University of Antwerp, Antwerp 2610, Belgium
}

(Received 31 May 2016; accepted 7 August 2016; published online 1 September 2016)

\begin{abstract}
Iridium (Ir) modified Silicon (Si) (001) surface is studied with Scanning Tunneling Microscopy/ Spectroscopy (STM/STS) and Density Functional Theory (DFT). A model for Ir-silicide nanowires based on STM images and ab-initio calculations is proposed. According to our model, the Ir adatom is on the top of the substrate dimer row and directly binds to the dimer atoms. I-V curves measured at $77 \mathrm{~K}$ shows that the nanowires are metallic. DFT calculations confirm strong metallic nature of the nanowires. Published by AIP Publishing. [http://dx.doi.org/10.1063/1.4961550]
\end{abstract}

\section{INTRODUCTION}

Building nanostructures with unique electronic properties on flat and/or vicinal Si (silicon) and Ge (germanium) substrates have been the goal of many researchers. ${ }^{1-7}$ Since these substrates are mainly used in semiconductor industry, they can provide a smooth transition from a prototype in a research lab to a consumer product. Some of these structures have high aspect ratios; therefore, they are called nanowires. Nanowires may exhibit interesting phenomena such as quantum confinement, ${ }^{8,9}$ Peierls instability, ${ }^{10-12}$ and Luttinger liquid. ${ }^{13}$ They can be made up of various elements ranging from bismuth ${ }^{14}$ and rare-earth metals ${ }^{15-17}$ to transition metals. ${ }^{18,19}$ Among those, transition metal nanowires have a special place because of the fact that they do not react with air to form insulating oxide layers. Ir (iridium) has already been shown to form nanowires on Ge (001), Si (001), and Si (110) surfaces. $^{20-22}$

In this paper, we report Scanning Tunneling Microscopy/ Spectroscopy (STM/STS) data to reveal electronic properties of Ir-silicide nanowires alongside with ab-initio density functional theory (DFT) calculations to explain the origins of these electronic properties and the structure of Ir-silicide nanowires.

\section{EXPERIMENT}

We purchased Ir (99.9\%) wire from Goodfellow. We used a p-type and B-doped Si (001) substrate. The samples were mounted on molybdenum holders, and contact of the samples to any other metal during preparation and experiment was carefully avoided. The ultra-high vacuum (UHV) system is equipped with Omicron variable temperature Scanning Tunneling Microscopy (STM) and LK technologies RVL2000 Low energy electron diffraction (LEED)Auger spectrometer system. The base pressure of the system was kept below $5 \times 10^{-10}$ mbar. Before introducing $\operatorname{Si}(001)$ samples in the UHV chamber, samples were dipped in isopropanol and dried with $N_{2}$ gas. $\operatorname{Si}(001)$ samples were

\footnotetext{
a) Author to whom correspondence should be addressed. Electronic addresses: gulseren@fen.bilkent.edu.tr and nuri.oncel@und.edu
}

degassed in the vacuum for at least two days prior to flashannealing cycles at $1250{ }^{\circ} \mathrm{C}$. The quality of the clean $\mathrm{Si}(001)$ samples was checked and confirmed both with LEED and STM before depositing Ir. 0.25 monolayer of Ir was deposited over the clean $\mathrm{Si}(001)$ surfaces from a direct current heated Ir wire. After deposition at room temperature, the sample was annealed at $700^{\circ} \mathrm{C}$. The sample temperature was measured with a pyrometer.

\section{THEORY}

All calculations were performed within plane-wave pseudopotential method based on density functional theory as implemented in the Vienna $A b$-initio Simulation package (VASP). ${ }^{23}$ We described the ions by employing PAW pseudopotential for atomic core region. ${ }^{24}$ The generalized gradient approximation was used for the exchange-correlation functional. Plane wave basis set was used for the expansion of the Kohn-Sham orbitals. After the extensive test calculations for the total energy convergence, the kinetic energy cut-off for plane wave expansion was set to $400 \mathrm{eV}$. The Brillouin zone (BZ) of the silicon surface is sampled with a k-mesh of $10 \times 10 \times 1$ within Monkhorst-Pack scheme. ${ }^{25}$ A vacuum region of $15 \AA$ is introduced in order to prevent spurious interaction between the periodic slabs. In order to minimize the interaction of silicon surfaces at the bottom and top of the slab structure, we determined the number of layers by observing relaxation shift of the first layer atom positions. Test calculations revealed that nine silicon layers were sufficient to describe $\mathrm{Si}(001)$ surface accurately. Four bottom layers were fixed at their corresponding bulk atomic positions in the calculations. Moreover, hydrogen atoms were attached to all silicon atoms at the bottom layer in order to saturate the dangling bonds. Thus, we obtained a system resembling the Si-bulk crystal. Except the atoms in the four lower layers, all the other atoms were allowed to relax and reconstruct.

\section{RESULTS}

The LEED and STM measurements showed that if annealed at $700{ }^{\circ} \mathrm{C}$, Ir-modified $\mathrm{Si}(001)$ surface turned into 
relatively small, irregular-shaped Ir-silicide terraces that exhibit a $p(2 \times 2)$ LEED pattern. If the sample was annealed above $800^{\circ} \mathrm{C}$, these terraces disappeared and $\mathrm{Si}(001)$ surface started to come back. The nanowires on the terraces stretch along [110] directions similar to dimer rows of a pristine $\mathrm{Si}(001)$ surface. $^{21,26}$ This indicates that the morphology of $\mathrm{Si}(001)$ surface directly influences the structure of these nanowires. The surface has Ir-rich terraces surrounded by $\mathrm{Si}(001)$ terraces with a high density of vacancy line defects (see Figure 1(a)). Figures 1(b) and 1(c) show high resolution STM images of Ir-rich terraces.

In order to study the electronic properties of the nanowires, we performed I-V measurements at $77 \mathrm{~K}$ (see Figure 2(a)). We calculated dI/dV curves numerically from the measured I-V curves. We did not see any difference between the

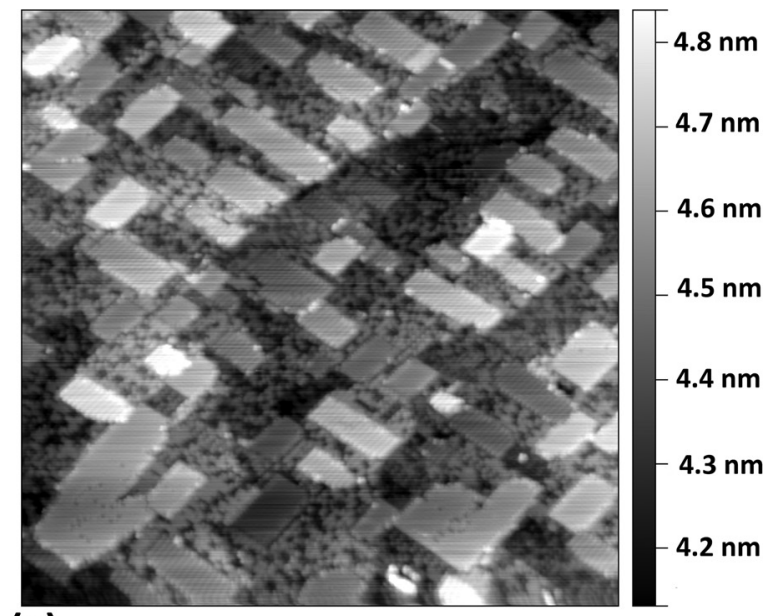

(a)

(b)

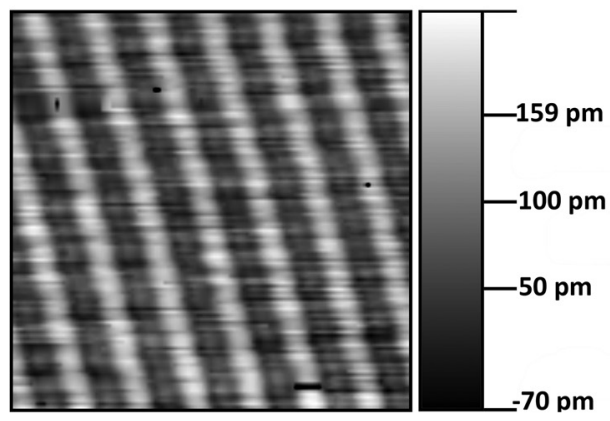

(c)

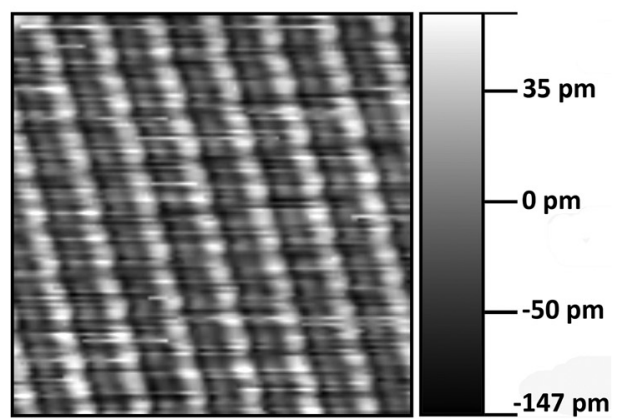

FIG. 1. (a) A $100 \mathrm{~nm} \times 100 \mathrm{~nm}$ STM image of Ir modified Si(001) surface. The sample bias and the tunneling current are $-1.74 \mathrm{~V}$ and $0.43 \mathrm{nA}$, respectively. (b) and (c) are $6 \mathrm{~nm} \times 6 \mathrm{~nm}$ STM image of Ir-silicide nanowires. The images are from the same region recorded at the same time during forward and backward scanning. The scanning voltages are $0.619 \mathrm{~V}$ and $-0.658 \mathrm{~V}$, respectively. The tunneling current is $0.49 \mathrm{nA}$.
$\mathrm{dI} / \mathrm{dV}$ curves measured on the nanowires and in the trough between the nanowires. $\mathrm{dI} / \mathrm{dV}$ curves measured on the nanowires have nonzero conductance around the Fermi level, indicating that the nanowires are metallic. There are three well-resolved peaks located at about $-0.57 \mathrm{~V},-0.15 \mathrm{~V}$, and $0.50 \mathrm{~V}$. On the other hand, the $\mathrm{dI} / \mathrm{dV}$ curves measured on the $\mathrm{Si}(001)$ surface by the Ir-silicide terraces have a band gap of about $0.5 \mathrm{eV}$ which is in line with the previously reported data on $\mathrm{Si}(001){ }^{27}$

We investigated the interaction of a single Ir atom with a $2 \times 2 \mathrm{Si}(001)$ surface by placing the Ir atom on various positions on the surface. We chose five possible adsorption sites at the surface (see Figure 3). The binding energy of Ir atom was calculated using the following expression:

$$
E_{\text {Binding }}=\left(E_{\text {total }}(\operatorname{Ir}+\text { slab })-E_{\text {total }}(\text { slab })-N \cdot E_{\text {Ir }}\right) / N,
$$

where $E_{\text {total }}(I r+$ slab $)$ and $E_{\text {total }}($ slab $)$ are the total energies of the Si slab with and without Ir atom, respectively. Here, $E_{I r}$ is the total energy of a single Ir atom. The possible adsorption sites were labeled with capital letters from A to E. The relative binding energy $\left(E_{\text {Binding }}\right)$ with respect to the ground state structure for all these five possible adsorption sites was listed in the table. The most stable adsorption site was obtained when an Ir atom was placed on a hollow region between dimer rows (B-site). After relaxing the structure, Ir atom dives into the bulk and it tends to merge into the fourth layer of the slab indicating that the Ir atom wants to bind as many $\mathrm{Si}$ atoms as possible. At this lowest energy configuration, Ir binds to six surface and subsurface Si atoms. On the other hand, for all the other possible adsorption sites, the Ir atom binds to at most four $\mathrm{Si}$ atoms. Although the sites $\mathrm{A}$ and $\mathrm{B}$ are the two lowest binding energy sites, they do not provide a suitable configuration to form the nanowires because of the surface reconstruction. The site $\mathrm{C}$, where the Ir atom occupies a hollow position between neighboring $\mathrm{Si}$ dimers, has the third lowest binding energy. The relaxed structure for the site $\mathrm{C}$ is depicted in Figure 4. Due to the strong interaction, as in the case of pristine $\mathrm{Si}(001)$ surface, $\mathrm{Si}$ dimers are flipped with respect to each other. We find two different Ir-Si bond lengths, which are 2.42 and $2.45 \AA$. The height of the Ir atom from the plane formed by the dimer atoms is about $1.1 \AA$. The site D is apparently not a stable adsorption site, and the temperature effects are expected to hamper the occupation of this position.

Considering both the calculations on the adsorption of a single Ir atom and measured STM images, we came up with a model of Ir-silicide nanowires (see Figure 4). The local bonding environment of the $\mathrm{Ir}$ atom on the site $\mathrm{C}$ is similar to the fluorite structure of $\mathrm{IrSi}_{2}$, where $\mathrm{Ir}$ atoms form a face-centered cell and $\mathrm{Si}$ atoms occupy the eight interstitial tetrahedral sites (see Figure 5). ${ }^{28}$ As depicted in Figure 5(a), the Ir atom directly binds to $\mathrm{Si}_{1}, \mathrm{Si}_{2}, \mathrm{Si}_{3}$, and $\mathrm{Si}_{4}$ atoms, which form a square pyramid. In the case of $\mathrm{Si}$ (001) surface, we observe a similar pyramid structure with a nonplanar base formed by $\mathrm{Si}_{1}, \mathrm{Si}_{2}, \mathrm{Si}_{3}$, and $\mathrm{Si}_{4}$ atoms of dimers (see Figure 5(b)), suggesting that at high Ir concentrations, it is possible to grow a fluorite-type $\mathrm{IrSi}_{2}$ structure on the Si (001) surface. 

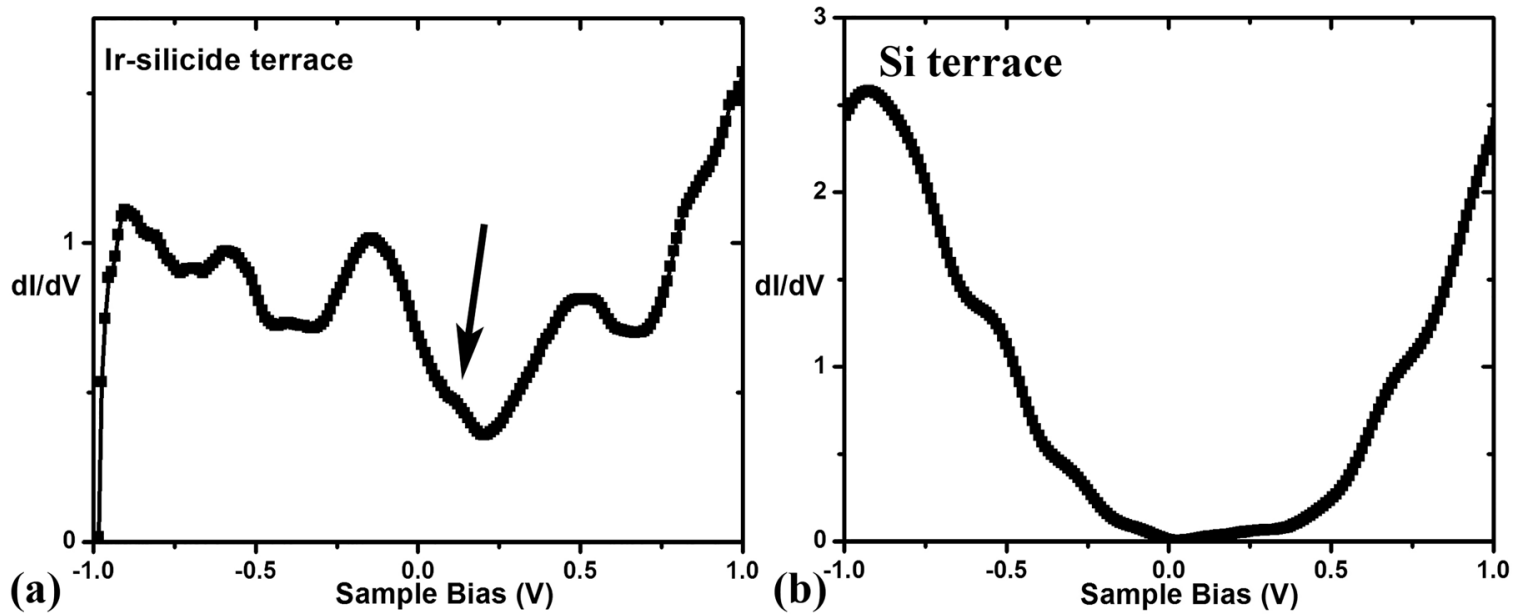

FIG. 2. (a) and (b) are $\mathrm{dI} / \mathrm{dV}$ curves measured on Ir-silicide and $\mathrm{Si}(001)$ terrace, respectively.

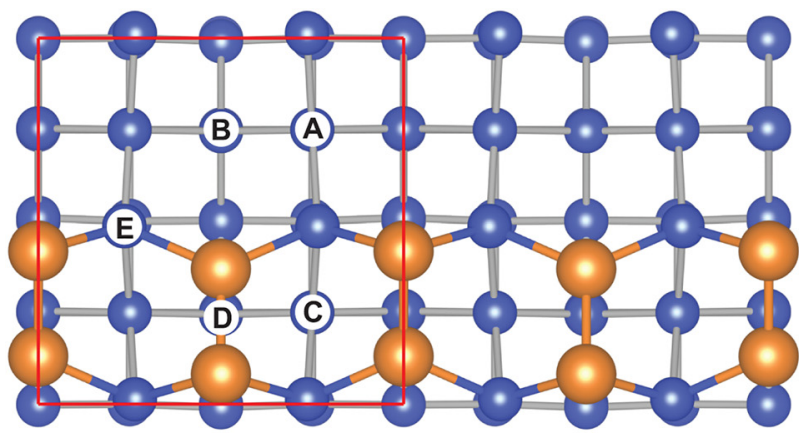

\begin{tabular}{|c|c|}
\hline $\begin{array}{c}\text { Adsorption } \\
\text { site }\end{array}$ & $\begin{array}{c}\text { Relative } \\
\text { Energy }(\mathrm{eV})\end{array}$ \\
\hline A & 0.42 \\
\hline B & 0 \\
\hline C & 0.53 \\
\hline D & 1.18 \\
\hline E & 0.57 \\
\hline
\end{tabular}

FIG. 3. On the left, possible adsorption sites for an $\mathrm{Ir}$ atom on $\mathrm{Si}(001)$ surface are shown on a model of the $\mathrm{Si}(001)$ surface. Letters A to E refer to the possible adsorption sites. The table on the right shows relative binding energies of the Ir atom at various adsorption sites with respect to the ground state structure (i.e., the site B).

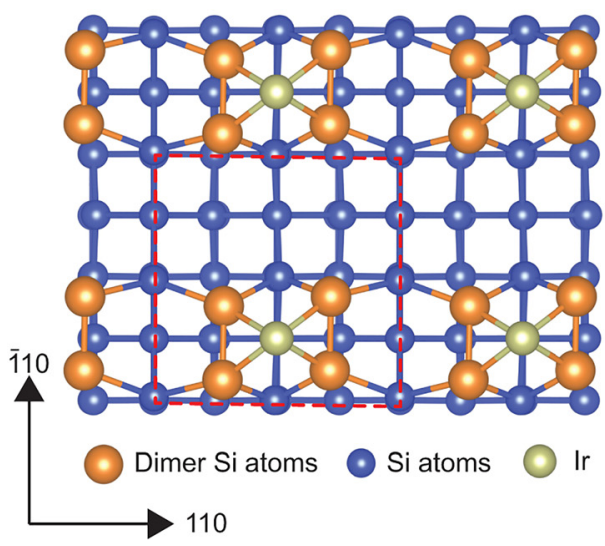

(a)

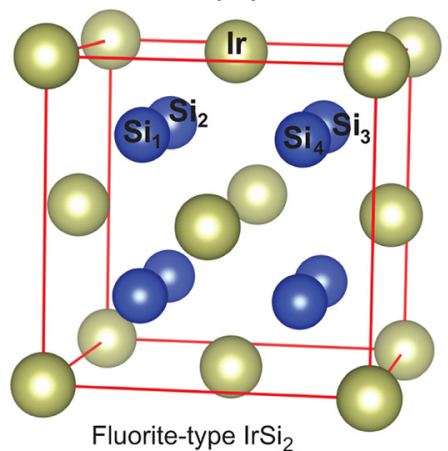

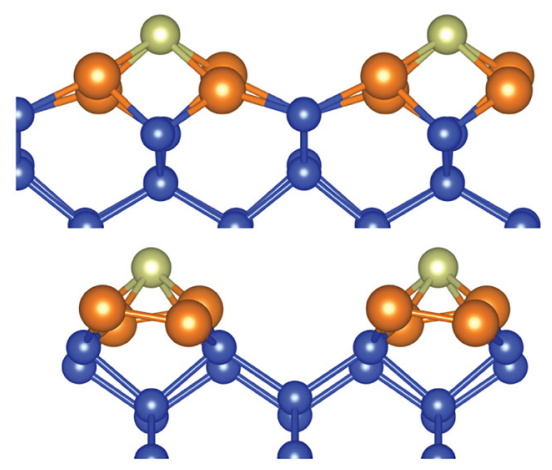

(b)

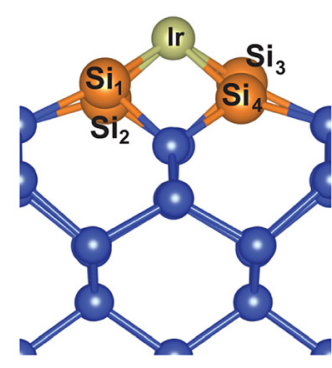

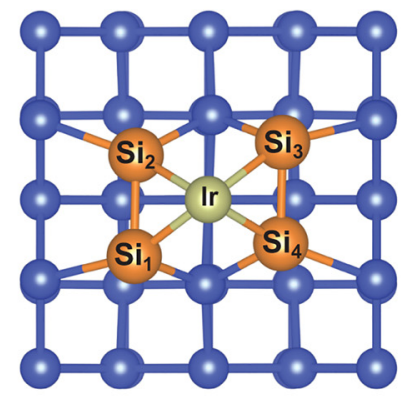

FIG. 4. Proposed model of Ir-silicide nanowires on $\mathrm{Si}(001)$ surface. Left (right) is a top (side) view of the proposed surface. Red dashed lines enclose a $2 \times 2$ surface cell.
FIG. 5. (a) Bulk structure of fluoritetype $\mathrm{IrSi}_{2}$ and (b) side and top views of local environment of the Ir atom on the $\mathrm{Si}(001)$ surface. 


\section{DISCUSSION}

In order to compare with measured $\mathrm{dI} / \mathrm{dV}$ curves, we calculated DOS curves for the proposed model (see Figure $6)$. The model predicts that the surface is metallic and there are three well-resolved peaks around the Fermi level. Similarly, the fluorite-type $\mathrm{IrSi}_{2}$ also exhibits metallic behavior. $^{28}$ A quick comparison reveals that the experimental dI/ $\mathrm{dV}$ curves and the calculated DOS curves show remarkable similarities. Both have states located at about $-0.55 \mathrm{eV}$, $-0.13 \mathrm{eV}$, and $0.46 \mathrm{eV}$, indicating that the proposed model accurately reproduces the measured electronic properties of the system. A well-defined peak right above the Fermi level in the calculated DOS graph is vaguely visible in the experimental $\mathrm{dI} / \mathrm{dV}$ curves (indicated by an arrow). We attribute the vagueness of this peak to the broadening of the STS peaks at finite temperature. In order to find out the origins of the observed states, we also calculated projected DOS curves for the dimer atoms and Ir atom of the surface (see Figure 6). Since the Ir atom sits on the top dimer row, its contribution to the DOS, especially, below the Fermi level is significant. The state at $0.46 \mathrm{eV}$ has almost equal contributions from both $\mathrm{Si}$ dimers and $\mathrm{Ir}$ atom. The calculated DOS points out a strong interaction between $\mathrm{Si}$ and $\mathrm{Ir}$ atoms and hybridization between Si $p$ states and Ir $d$ states. In order to correlate the proposed model with the measured STM images, we also calculated STM images for bias voltages of $-0.658 \mathrm{~V}$ and $+0.618 \mathrm{~V}$ (see Figure 7). Unlike experimental data shown in Figures 1(b) and 1(c), the calculated STM images of the surface have ellipsoid peaks. Since the Ir atom is closer to the STM tip, it dominates the calculated STM images. Si dimers have significant DOS around Fermi level, but they are located further away from the STM tip and their contribution to the tunneling current is small. However, the Si dimers are responsible for the elliptical shape of the peaks. For the negative and positive bias voltages, the major and minor axes of ellipses in the calculated STM images are swapped, showing that upper and lower dimer atoms have different densities for occupied and unoccupied states around Fermi level. In measured STM images (see Figures 1(b) and 1(c)), the direction of the major axis of the ellipsoid is independent of the DOS sample bias and it is along the dimer row direction. We attribute this to the fact that in the theoretical model, the $\mathrm{Si}$ dimers are fixed; however in reality, the dimers can flip. It is

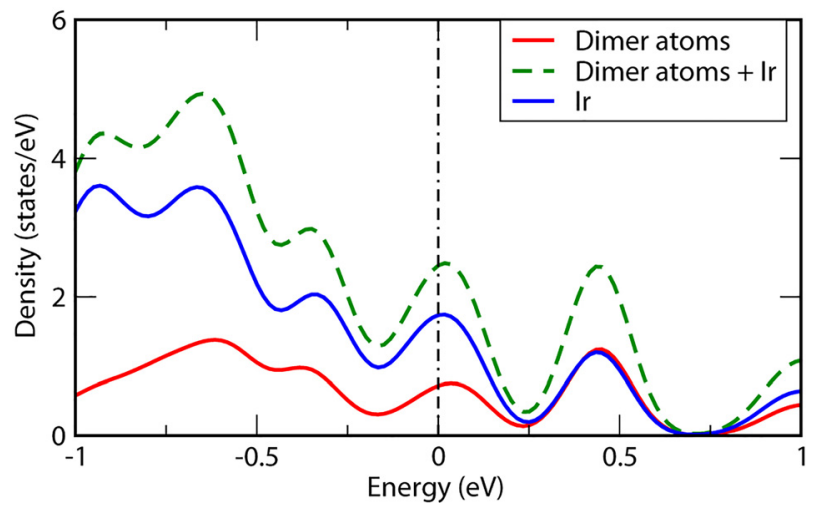

FIG. 6. Calculated projected DOS curves of the proposed model. (a)

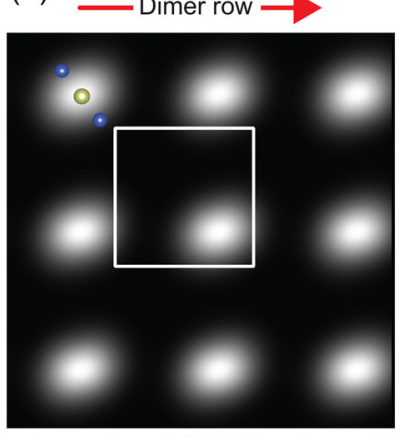

$-0.658 \mathrm{~V}$

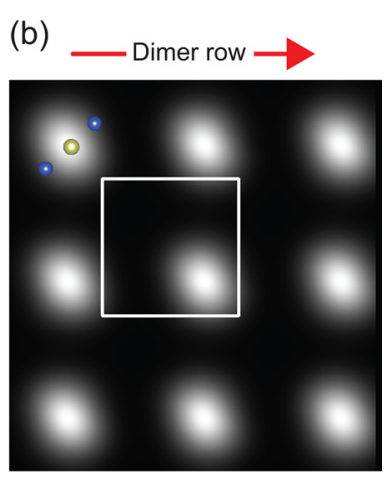

(c)

$+0.618 \mathrm{~V}$

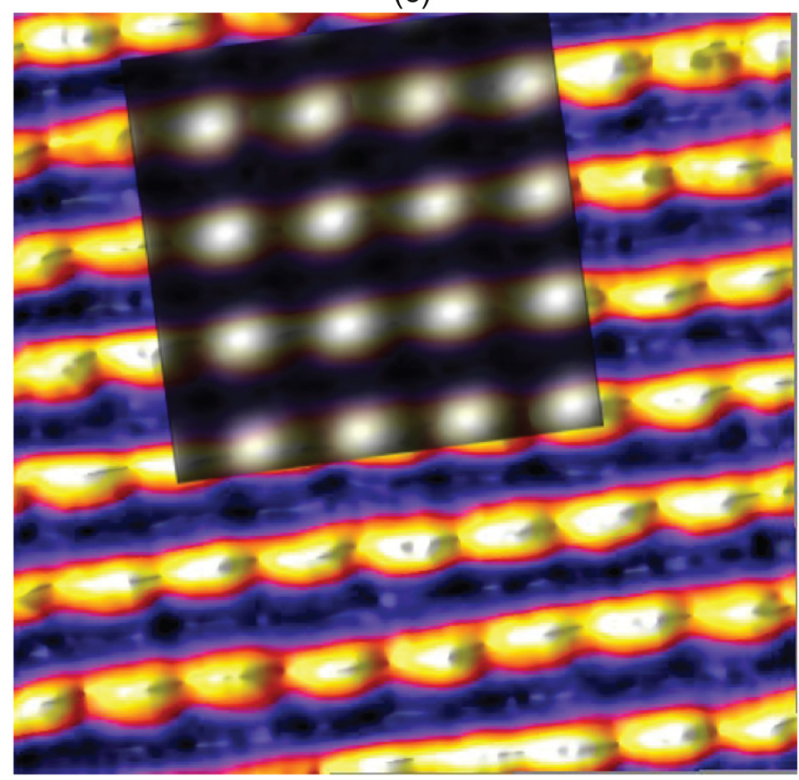

FIG. 7. Simulated STM image for bias voltage of (a) $-0.658 \mathrm{~V}$ and (b) $0.618 \mathrm{~V}$. Blue atoms mark the dimer Si atoms that do not contribute STM images. White squares enclose a $2 \times 2$ surface cell. The red arrows show the direction of dimer row. (c) Background: A $15 \mathrm{~nm} \times 15 \mathrm{~nm}$ STM image of Irsilicide nanowires, sample bias, and tunneling current are $-0.658 \mathrm{~V}$ and $0.68 \mathrm{nA}$. The inset is the calculated STM image.

known that on pristine $\mathrm{Si}(001)$ surface, at temperatures above $\sim 200 \mathrm{~K}$, Si dimers constantly flip which causes dimers appearing as oblong protrusions in measured STM images leading to characteristic $2 \times 1$ structure. $^{29}$ A similar flipping motion would lead to an STM image that sees the averaged DOS on dimer atoms and tunneling through these flipping dimers makes the peak elongate along the dimer row direction.

\section{CONCLUSION}

In conclusion, we studied the structural and electronic properties of Ir-silicide nanowires with STM/STS and DFT. The Ir atoms are found to sit at the top of the dimer row. DOS calculations and measured $\mathrm{dI} / \mathrm{dV}$ curves show that the nanowires are metallic. Both measured and calculated DOS curves show that there are three well-resolved states around the Fermi level. The states at $-0.55 \mathrm{eV}$ and $-0.13 \mathrm{eV}$ carry large contributions from Ir atoms; however, state at $0.46 \mathrm{eV}$ has equal contribution from Si dimers and Ir atom. While the Ir atoms mainly dominate the STM images, Si dimers also have noticeable contribution. 


\section{ACKNOWLEDGMENTS}

We gratefully acknowledge the NSF (Grant No. DMR1306101) for financial support. Computational resources were provided by TUBITAK ULAKBIM, High Performance and Grid Computing Center (TR-Grid e-Infrastructure), and HPC infrastructure of the University of Antwerp (CalcUA), a division of the Flemish Supercomputer Center (VSC), which is funded by the Hercules foundation.

${ }^{1}$ Y. P. Zhang, L. Yang, Y. H. Lai, G. Q. Xu, and X. S. Wang, Appl. Phys. Lett. 84, 401 (2004).

${ }^{2}$ H. Okino, I. Matsuda, R. Hobara, Y. Hosomura, S. Hasegawa, and P. A. Bennett, Appl. Phys. Lett. 86, 233108 (2005).

${ }^{3}$ A. Kida, H. Kajiyama, S. Heike, T. Hashizume, and K. Koike, Appl. Phys. Lett. 75, 540 (1999).

${ }^{4}$ N. de Jong, R. Heimbuch, S. Eliëns, S. Smit, E. Frantzeskakis, J.-S. Caux,

H. J. W. Zandvliet, and M. S. Golden, Phys. Rev. B. 93, 235444 (2016)

${ }^{5}$ J. R. Ahn, H. W. Yeom, H. S. Yoon, and I.-W. Lyo, Phys. Rev. Lett. 91, 196403 (2003).

${ }^{6}$ D. Nicholls and N. Oncel, J. Phys. Condens. Matter 25, 445004 (2013).

${ }^{7}$ N. Oncel, D. Cakir, J. H. Dil, B. Slomski, and G. Landolt, J. Phys. Condens. Matter 26, 285501 (2014).

${ }^{8}$ N. Oncel, A. van Houselt, J. Huijben, A. S. Hallback, O. Gurlu, H. J. W. Zandvliet, and B. Poelsema, Phys. Rev. Lett. 95, 116801 (2005).

${ }^{9}$ A. van Houselt, N. Oncel, B. Poelsema, and H. J. W. Zandvliet, Nanoletters 6, 1439 (2006).

${ }^{10}$ R. Losio, K. N. Altmann, and F. J. Himpsel, Phys. Rev. Lett. 85, 808 (2000).

${ }^{11}$ J. L. McChesney, J. N. Crain, V. Perez-Dieste, F. Zheng, M. C. Gallagher, M. Bissen, C. Gundelach, and F. J. Himpsel, Phys. Rev. B 70, 195430 (2004).
${ }^{12}$ A. van Houselt, T. Gnielka, J. M. J. Aan de Brugh, N. Oncel, D. Kockmann, R. Heid, K.-P. Bohnen, B. Poelsema, and H. J. W. Zandvliet, Surf. Sci. 602, 1731 (2008).

${ }^{13}$ C. Blumenstein, J. Schäfer, S. Mietke, S. Meyer, A. Dollinger, M. Lochner, X. Y. Cui, L. Patthey, R. Matzdorf, and R. Claessen, Nat. Phys. 7, 776 (2011).

${ }^{14}$ J. H. G. Owen, K. Miki, and D. R. Bowler, J. Mater. Sci. 41, 4568 (2006).

${ }^{15}$ Y. Chen, D. A. A. Ohlberg, G. Mederios-Ribario, Y. A. Chang, and R. S. Williams, Appl. Phys. Lett. 76, 4004 (2000).

${ }^{16}$ Y. Chen, D. A. A. Ohlberg, and R. S. Williams, J. Appl. Phys. 91, 3213 (2002).

${ }^{17}$ J. Nogami, B. Z. Liu, M. V. Katkov, C. Ohbuchi, and N. O. Birge, Phys. Rev. B 63, 233305 (2001).

${ }^{18}$ O. Gurlu, O. A. O. Adam, H. J. W. Zandvliet, and B. Poelsema, Appl. Phys. Lett. 83, 4610 (2003).

${ }^{19}$ J. Wang, M. Li, and E. I. Altman, Phys. Rev. B 70, 233312 (2004).

${ }^{20}$ T. F. Mocking, P. Bampous, N. Oncel, B. Poelsema, and H. J. W. Zandvliet, Nat. Commun. 4, 2387 (2013).

${ }^{21}$ N. Oncel and D. Nicholls, J. Phys. Condens. Matter 25, 014010 (2013).

${ }^{22}$ R. N. Mohottige and N. Oncel, Surf. Sci. 641, 237 (2015).

${ }^{23}$ G. Kresse and J. Furthmuller, Comput. Mater. Sci. 6, 15 (1996); Phys. Rev. B 54, 11169 (1996).

${ }^{24}$ P. E. Blochl, Phys. Rev. B 50, $17953-17979$ (1994); G. Kresse and D. Joubert, ibid. 59, 1758-1775 (1999).

${ }^{25}$ H. J. Monkhorst and J. D. Pack, Phys. Rev. B 13, 5188 (1976).

${ }^{26}$ H. J. W. Zandvliet, Rev. Mod. Phys. 72, 593 (2000).

${ }^{27}$ V. A. Ukraintsev, Z. Dohnalek, and J. T. Yates, Jr., Surf. Sci. 388, 132 (1997).

${ }^{28}$ U. Hormann, T. Remmele, J. E. Klepeis, O. Pankratov, H. Grunleitner, M. Schulz, M. Falke, and A. Bleloch, Phys. Rev. B. 79, 104116 (2009).

${ }^{29}$ T. Yokoyama and K. Takayanagi, Phys. Rev. B 61, R5078 (2000). 